\title{
Barriers to Colon Cancer Screening in South and South East Asia
}

\author{
Furqaan Ahmed ${ }^{1 *}$ and Khawar Mehdi ${ }^{2}$ \\ ${ }^{1}$ South City Hospital, Karachi, Pakistan \\ ${ }^{2}$ Getz Pharma (Pvt.) Ltd, Pakistan
}

Received: March 02, 2015; Accepted: May 11, 2015; Published: May 20, 2015

*Corresponding author: Furqaan Ahmed, South City Hospital, 128C KDA Scheme \#1, Karachi, Pakistan, 75350, E-mail: furqaan@hotmail.com

\begin{abstract}
Background: Colorectal cancer (CRC) screening has been shown to reduce the incidence of CRC and is now standard of care in the West.
\end{abstract}

Aim: To assess CRC screening practices among gastroenterologists in South and South East Asia.

Methods: Gastroenterologists from South and South East Asia attending the International Gastroenterology and Hepatology Forum in Yangon, Myanmar in November 2012 were surveyed regarding their CRC screening practices.

Results: The 67 gastroenterologists were from the following countries: Pakistan 24, Sri Lanka 2, Myanmar 24, Philippines 9, Laos 4, and Cambodia 4. National programs for CRC screening do not exist in any of the countries studied. The incidence of CRC is not known in any of these countries. The following obstacles to CRC screening in their countries were cited: lack of awareness about CRC $60 \%$, lack of resources $70 \%$, lack of local data about CRC $28 \%$, and lack of patient acceptance for CRC screening $27 \%$. Of those who said that lack of resources was an obstacle to CRC screening in their country, the lack of the following resources were cited: money $61 \%$, doctors $17 \%$, and facilities $30 \%$. $31 \%$ of the gastroenterologists surveyed practiced some of form CRC screening in their own practices.

Conclusions: Based on this sample of gastroenterologists representing six countries from the region, there appears to be limited CRC screening in South and South East Asia. Issues relating to lack of local data, lack of awareness, and lack of resources (money, trained doctors, facilities) limit CRC screening in this region.

\section{Introduction}

Colorectal cancer (CRC) is the fourth leading cause of cancer death worldwide [1]. CRC screening has been shown to reduce the incidence of CRC [2-5]. CRC screening is now standard of care in the West and is endorsed by, amongst others, the United States Preventive Services Task Force and the Council of the European Union $[6,7]$.

There are very limited data pertaining to CRC and CRC screening from South and South East Asia. The objective of this study was to assess CRC screening practices among gastroenterologists in South and South East Asia.

\section{Methods}

This study was conducted by surveying gastroenterologists from South and South East Asia attending the International Gastroenterology and Hepatology Forum (IGHF) in Yangon, Myanmar in November 2012. The IGHF is a forum created to bring together Gastroenterology and Hepatology faculty, practitioners, and trainees from different countries in the region on a single platform to exchange ideas, learn from one another, and build ties in order to improve the state of gastrointestinal and liver healthcare and research in their respective countries.

Using a questionnaire, demographic and practice date were collected including age, gender, type of practice setting and number of years in practice. Gastroenterology trainees were excluded. Questions were asked about national CRC screening programs, local CRC epidemiological data, and personal CRC screening practices. Finally, the gastroenterologists were questioned regarding perceived barriers to CRC screening in their respective countries.

The study was approved by our Institutional Review Board.

\section{Results}

67 gastroenterologists were included in this study. 69\% (46) were male. 69\% (46) were in academic practice. The doctors were from the following countries: Pakistan 24 (36\%), Sri Lanka 2 (3\%), Myanmar 24 (36\%), Philippines 9 (13\%), Laos 4 (6\%), and Cambodia $4(6 \%)$. The doctors have been in practice for a time ranging from 1 to 30 years.

National programs for CRC screening do not exist in any of the countries studied. Furthermore, the incidence of CRC is not known in any of these countries. 31\% (20) of the gastroenterologists surveyed practiced some form of CRC screening in their own practices. The modalities used include fecal occult blood testing (FOBT) by 2, sigmoidoscopy (1), colonoscopy (12), and a combination of FOBT and colonoscopy (5). The frequency of screening varied widely and ranged from 1-50 years.

When asked about obstacles to CRC screening in their countries, the following reasons were cited: 1 . Lack of awareness about CRC $60 \%$ (40), 2. Lack of resources 70\% (47), 3. Lack of 
local epidemiologic data on CRC 28\% (19), and 4. Lack of patient acceptance for CRC screening $27 \%$ (18). Of those who said that lack of resources was an obstacle to CRC screening in their country, the lack of the following resources were cited: 1 . Money $61 \%$ (29), 2. Doctors 17\% (8), and 3. Facilities 30\% (20).

\section{Discussion}

This study shows that the South and South East Asia region has fallen behind global trends in colorectal cancer screening. National programs for CRC screening do not exist in the countries surveyed in this study. Epidemiologic data is lacking or nonexistent. Multiple obstacles have been identified in this study including lack of local data, awareness, and resources.

A basic prerequisite for any screening program is knowledge of the incidence and prevalence of the disease in question. Only after knowing the incidence and prevalence of a particular cancer can one consider screening practices and then study the effects of such interventions. Without basic epidemiologic data, any screening program is unjustifiable because the benefits of such programs would be untestable. The gastroenterologists surveyed in this study report a lack of basic epidemiologic data regarding $\mathrm{CRC}$ in the region.

Gastroenterologists and Gastroenterology societies in these countries need to make a strong effort to conduct epidemiologic studies to gather this data, after which they can develop guidelines for screening that meet local requirements, taking into account local resources. The Asia Pacific Working Group on Colorectal Cancer is trying to promote CRC screening in the Asia Pacific region by publishing data on adenoma frequency and by developing CRC screening guidelines [8,9]. Unfortunately, in both published colonoscopy polyp studies and guidelines, South Asia is not represented and South East Asia is underrepresented. It is hoped that their future projects will rectify this.

Apart from the lack of epidemiologic data, other identified obstacles to CRC screening in the region include a lack of awareness about CRC, lack of resources, and a lack of patient acceptance for CRC screening. Each of these obstacles requires further study. For example, in the United States, studies have shown that women of Chinese origin and women of Muslim faith demonstrate resistance to CRC screening but for different reasons $[10,11]$.

Based on this sample of gastroenterologists representing six countries from the region, there appears to be limited CRC screening in South and South East Asia. Issues relating to lack of local data, lack of awareness, and lack of resources (money, trained doctors, facilities) limit CRC screening in this region.

\section{References}

1. Parkin DM, Bray F, Ferlay J, Pisani P. Estimating the world cancer burden: Globocan 2000. Int J Cancer. 2001;94:11668491.

2. Baxter NN, Goldwasser MA, Paszat LF, Saskin R, Urbach DR, Rabeneck L. Association of colonoscopy and death from colorectal cancer. Ann Intern Med. 2009;150:19075198.

3. Hardcastle JD, Chamberlain JO, Robinson MH, Moss SM, Amar SS, Balfour TW, et al. Randomised controlled trial of faecal-occultblood screening for colorectal cancer. Lancet. 1996;348:8942775. doi:10.1016/S0140-6736(96)03386-7.

4. Mandel JS, Bond JH, Church TR, Snover DC, Bradley GM, Schuman LM, et al. Reducing mortality from colorectal cancer by screening for fecal occult blood. Minnesota Colon Cancer Control Study. N Engl J Med. 1993;328:8474513. doi:10.1056/NEJM199305133281901.

5. Kronborg O, Fenger C, Olsen J, Jørgensen OD, Søndergaard O. Randomised study of screening for colorectal cancer with faecaloccult-blood test. Lancet. 1996;348:8942774. doi:10.1016/S01406736(96)03430-7.

6. U.S. Preventive Services Task Force. Screening for colorectal cancer: U.S. Preventive Services Task Force recommendation statement. Ann Intern Med. 2008;149:18838716.

7. European Council. Council recommendation of 2 December 2003 on cancer screening (2003/878/EC). OJ L 327 (16 December 2003),3,1415.

8. Sung JJY, Lau JYW, Young GP, Sano Y, Chiu HM, Byeon JS, et al. Asia Pacific consensus recommendations for colorectal cancer screening. Gut. 2008;57:18628378. doi:10.1136/gut.2007.146316.

9. Byeon J-S, Yang S-K, Kim TI, Kim WH, Lau JYW, Leung W-K, et al. Colorectal neoplasm in asymptomatic Asians: a prospective multinational multicenter colonoscopy survey. Gastrointest Endosc. 2007;65:17531636. doi:10.1016/j.gie.2006.12.065.

10. Wang JH-Y, Liang W, Chen M-Y, Cullen J, Feng S, Yi B, et al. The influence of culture and cancer worry on colon cancer screening among older Chinese-American women. Ethn Dis. 2006;16:17682242.

11. Padela AI, Rodriguez del Pozo P. Muslim patients and cross-gender interactions in medicine: an Islamic bioethical perspective. J Med Ethics. 2011;37:21041237. doi:10.1136/jme.2010.037614. 